\title{
Numerical Definition of Indicators of the Development of Creeping Oil Layer on Values of Change of an Well Production
}

\author{
Kazymov Bunyad Zinhar Oglu \\ Candidate of Technical Sciences, Leading Researcher of Institute of Oil and Gas of National Academy of Sciences of Azerbaijan, Baku, \\ Azerbaijan
}

\author{
Email address: \\ bunyadkazymov1969@gmail.com
}

To cite this article:

Kazymov Bunyad Zinhar Oglu. Numerical Definition of Indicators of the Development of Creeping Oil Layer on Values of Change of an Well Production. International Journal of Theoretical and Applied Mathematics. Vol. 3, No. 5, 2017, pp. 167-173.

doi: $10.11648 /$ j.ijtam.20170305.13

Received: October 16, 2016; Accepted: April 25, 2017; Published: October 31, 2017

\begin{abstract}
For the last time have been saved up in the large volume of laboratory and experimental and actual data which, in the turn, confirm need of the accounting of nonlinear deformations of rocks at the solution of hydrogasdynamic problems of development of oil and gas pools. These deformations of rocks, generally have rheological character which can affect indicators of development of the deposits of oil and gas developed at various natural and technological modes noticeably. Considering this circumstance, article is devoted to the solution of a task the movement of the gas-cut oil to the well in oil layer which breeds are exposed to creeping deformation, on condition of the set well production. The numerical solution of a task has been received and on the basis of hypothetical model of layer the numerical experiment has been made. By results of numerical calculations character influence of creep of rocks on the defined indicators of development of the oil pool has been established.
\end{abstract}

Keywords: Reservoir Pressure, Porosity, Gas-Oil Ratio, Numerical Decision, Boundary Value Problem

\section{Introduction}

The results of experimental, theoretical and field studies show that collectors of deep oil and gas fields in the process of development by decreasing reservoir pressure under the influence of rock pressure undergo inelastic (relaxation and creeping) strains $[1,2]$. Therefore, consideration of these phenomena will improve the accuracy and reliability of the hydrodynamic calculations forecasting of technological parameters of development of this type of deposits [3].

It should be noted that taking into account the influence of the nonlinear deformation of rocks on the defined indicators of oil and gas deposits, basically, material balance method have been studied in most works, one of the authors, which is also the author of the article [3]. While focused on the study of relaxation deformation of rocks on the development of indicators. However, in some cases, tasks were considered in the light of the creeping deformation of rocks. Since the results of appropriate studies show that even use enough total relaxation dependencies between porosity and inside- formation pressure is not sufficient for describing the nonlinear deformation processes occurring in rocks during development of oil and gas, due to the fact that the expansion of this type of dependency may be hereditary. Because of this, it was necessary to use to describe the deformation processes in rocks other rheological relationship inherent creep of rocks.

Data of wells hydrodynamic research in many fields suggests that the actual time of full stabilization of incomplete restoration of reservoir pressure can reach several months and even more. However, the results of calculations in the framework of the theory of elastic regime shows that the estimated time of stabilization of the reservoir pressure are insignificant and much less than the actual. Specified is associated with the creeping deformation of the porous medium, which, in its turn, is associated with a long dive under the influence of static montain pressure. 


\subsection{About Models of Definition of Permeability and Porosity Depending on Pressure in Creeping Environments}

By I. M. Ametov and K. S. Basniyev [2] for the mathematical description of the relaxation phenomena at a filtration of liquid and gas in layers with creeping deformation have used representation of volume creep of rocks. According to these representations, permeability and porosity of rocks is described by the following equations:

$$
\begin{aligned}
& k(r, t)=k_{0}\left[1+k_{1} \int_{0}^{t} e^{-\frac{t-\tau}{\tau_{k}}}\left(p(r, \tau)-p_{0}\right) d \tau\right] \\
& m(r, t)=m_{0}\left[1+m_{1} \int_{0}^{t} e^{-\frac{t-\tau}{\tau_{m}}}\left(p(r, \tau)-p_{0}\right) d \tau\right]
\end{aligned}
$$

where $k_{0}, m_{0}$ - respectively initial permeability and porosity of layer; $m_{1}, k_{1}$ - creep parameters $\left(m_{1}=1 / \mu\right.$ - volume fluidity); $p_{0}$ - initial pressure of layer; $\tau_{k}$ and $\tau_{m}$ respectively time of a relaxation of permeability and porosity of the creeping environment.

Let's note that in (1) and (2) as a kernel of creep the fading exponential function of Voltaire has been used. Similar dependences of permeability (1) and porosity (2) can be received, using other functions of a kernel of creep. For example, for Abel's kernel, we have:

$$
\begin{aligned}
& k(r, t)=k_{0}\left[1+\delta_{k} \int_{0}^{t}(t-\tau)^{-\alpha}\left(p(r, \tau)-p_{0}\right) d \tau\right] \\
& m(r, t)=m_{0}\left[1+\delta_{m} \int_{0}^{t}(t-\tau)^{-\alpha}\left(p(r, \tau)-p_{0}\right) d \tau\right]
\end{aligned}
$$

where $\delta_{k}, \delta_{m}, \alpha$ - creep parameters, and $0<\alpha<1$.

Let's note that we offer the general dependence of the description of permeability and the porosity [3] different from these dependences. For example, for a kernel like Voltaire:

$$
\begin{aligned}
& k(r, t)=k_{0}\left[1+\alpha_{k}\left(p-p_{0}\right)+k_{1} \int_{0}^{t} e^{-\frac{t-\tau}{\tau_{k}}}\left(p-p_{0}\right) d \tau\right] \\
& m(r, t)=m_{0}\left[1+\beta_{r}\left(p-p_{0}\right)+m_{1} \int_{0}^{t} e^{-\frac{t-\tau}{\tau_{m}}}\left(p-p_{0}\right) d \tau\right] \\
& \frac{1}{r} \frac{\partial}{\partial r}\left(r \frac{\partial^{2} p^{2}}{\partial r^{2}}\right)=\frac{1}{\chi} \frac{\partial p^{2}}{\partial t}+\frac{2 p_{0} m_{1}}{\chi} \frac{\partial}{\partial t}\left[\int_{0}^{t} \exp \left(-\gamma_{m}(t-\tau)\right)\left(p^{2}-p_{0}^{2}\right) d \tau\right]
\end{aligned}
$$

where $\alpha_{k}$ and $\beta_{r}$ - respectively coefficients of elastic change of permeability and elastic compressibility of therock.

Solving jointly the continuity equations, the law of filtering, and also the specified ratios between porosity and permeability for each case it is possible to receive the differential equation of movement of the compressed liquid in layer which rocks are deformed by creep.

\subsection{The Short Analysis and the Characteristic of Works in the Chosen Direction of Researches}

Solving equation of continuity, the law of filtration, and also the relationship between porosity and permeability for each case to obtain the differential equation of motion of a compressible fluid in the reservoir, rocks which are deformed by creep. Following this, in [2] obtained the following equations for the description of motion of gas and liquid in a creeping environment, namely:

for compressible liquid:

$$
\begin{gathered}
\operatorname{div}\left\{\left[1+k_{1} \int_{0}^{t} \exp \left(-\gamma_{k}(t-\tau)\right)\left(p-p_{0}\right) d \tau\right] \nabla p\right\}=\frac{1}{\chi} \frac{\partial p}{\partial t}+ \\
+\frac{m_{1}}{\chi \beta_{l}} \frac{\partial}{\partial t}\left[\int_{0}^{t} \exp \left(-\gamma_{m}(t-\tau)\right)\left(p-p_{0}\right) d \tau\right]
\end{gathered}
$$

for gas:

$$
\begin{aligned}
& \operatorname{div}\left\{\left[1+\frac{k_{1}}{p_{0}} \int_{0}^{t} \exp \left(-\gamma_{k}(t-\tau)\right)\left(p^{2}-p_{0}^{2}\right) d \tau\right] \nabla p^{2}\right\}= \\
& =\frac{1}{\chi} \frac{\partial p^{2}}{\partial t}+\frac{2 p_{0} m_{1}}{\chi} \frac{\partial}{\partial t}\left[\int_{0}^{t} \exp \left(-\gamma_{m}(t-\tau)\right)\left(p^{2}-p_{0}^{2}\right) d \tau\right]
\end{aligned}
$$

where $\chi=\frac{k_{0}}{m_{0} \mu \beta_{l}}$ - in the case of liquid; $\chi=\frac{k_{0} \cdot p_{0}}{m_{0} \mu}$ - in the case of gas; $\gamma_{k}=1 / \tau_{k} ; \gamma_{m}=1 / \tau_{m} ; \mu$ and $\beta_{l}$ - respectively the viscosity and compressibility of the liquid.

Also, they solved the problem of filtering in creeping environments, and the comparison of the theoretical results with actual measurements on one of the specific wells gas condensate field and, eventually, the estimated time of creep of rocks under appropriate initial and boundary data. It was decided the differential equation of gas filtration in a creeping medium, the obtained from (8) at $k_{1}=0$ :
In recent years, started to develop work on the solution of such problems using numerical methods, the results of which are more important from the point of view of their use in engineering trade practice of field development. As well as 
developing work on the definition of filtration-capacitive and deformation parameters of oil and gas reservoirs, using the methods of diagnosing, curves of decline (or recovery) of the pressure in the wells, including identification methods [3-8].

A high level of reliability in forecasting of development of deposits, in addition to the integrity of a set of source data directly is determined by the methods of the study of appropriate water and gas flows of problems of a filtration of oil and gas in porous media with those or other technological conditions of wells that occur in the development of these deposits.

Original mathematical model of such type of problems are typically complex systems of differential equations, including physical and geological parameters of fluid and reservoir, as well as the dynamic production performance filtration of fluids in the formation. The study of the filtering process in the reservoir is provided by adding to this system of equations, appropriate initial and boundary conditions, which are chosen depending on the type of water and gas flows in the studied tasks.

With the increasing complexity of the investigated problem, adequately complicated procedure of selection of methods decisions, and therefore increase the error of the accuracy of the results the solution of these problems when using for this purpose the simplest mathematical apparatus. These circumstances preclude the use of a simple mathematical apparatus for the solution of sophisticated tasks, which is manifested in the development of oil and gas fields, even if incomplete accounting of the influence of complex deformation processes in rocks, and also at normal technological conditions of the wells and the reservoir.

Given the above comments, this article introduces a numerical solution of the filtration problem of the influx of aerated oil to the well in a creeping formation. The effect of creep of rocks is taken into account only through consideration of the functional relation between the porosity and formation pressure.

\section{Statement of the Problem and Its Solution}

A boundary value problem associated with filtration of gas-cut oil to the wellbore in oil deposits with creeping environment described:

- a system of differential and integral equations

$$
\begin{gathered}
\frac{1}{r} \frac{\partial}{\partial r}\left(\frac{\bar{k}_{o i l}(\sigma)}{\mu_{o i l}(p) a(p)} r \frac{\partial p}{\partial r}\right)=\frac{1}{K_{0}} \frac{\partial}{\partial t}\left(\frac{m \sigma}{a(p)}\right) \\
\frac{1}{r} \frac{\partial}{\partial r}\left(\left(\frac{\bar{k}_{o i l}(\sigma) s(p)}{\mu_{o i l}(p) a(p)}+\frac{\beta}{p_{a t}} \frac{\bar{k}_{\text {gas }}(\sigma) p}{\mu_{\text {gas }}(p)}\right) r \frac{\partial p}{\partial r}\right)= \\
=\frac{1}{K_{0}} \frac{\partial}{\partial t}\left\{m\left(\frac{s(p)}{a(p)} \sigma+\frac{\beta}{p_{a t}} p(1-\sigma)\right)\right\}
\end{gathered}
$$

$$
m=m_{0}\left[1+\beta_{r}\left(p-p_{0}\right)+m_{1} \int_{0}^{t} e^{-\gamma_{m}(t-\tau)}\left(p-p_{0}\right) d \tau\right]
$$

initial and boundary conditions

$$
\begin{gathered}
p(r, 0)=p_{0}, m(r, 0)=m_{0}, \sigma(r, 0)=1 \\
\left.\frac{2 \pi r h K_{0} \bar{k}_{\text {oil }}(\sigma)}{\mu_{\text {oil }}(p) a(p)} \frac{\partial p}{\partial r}\right|_{r=r_{w s}}=q_{\text {oil }}(t) \\
\left.\frac{\partial p}{\partial r}\right|_{r=r_{k}}=0
\end{gathered}
$$

where $p=p(r, t), m=m(r, t) ; p, m$ and $\sigma$ - respectively current reservoir pressure, porosity and oil saturation; $K_{0}$ absolute permeability; $q_{\text {oil }}$ - well production; $\bar{k}_{\text {oil }}$ and $\bar{k}_{\text {gas }}$ respectively, relative permeability of oil and gas; $a(p)$ and $s(p)$ - respectively the volumetric ratio of oil and gas solubility coefficient; $\mu_{\text {oil }}(p)$ and $\mu_{\text {gas }}(p)$ - respectively the viscosity of oil and gas; $\beta$ - temperature correction for the gas; $p_{a t}$ - atmospheric pressure; $r_{w}$ - well radius; $r_{k}$ drainage radius of a well; $r$ - radial coordinate $\left(r_{w} \leq r<r_{k}\right) ; t$ - development time $(t>0)$; other designations are given above.

The numerical solution of the problem (10) - (11) was obtained using the method with a sweep iterative refinement of nonlinear coefficients $[9,10]$. According to this method, the solution of a task in net area $\left\{\left(\xi_{i}, t_{j}\right): \xi_{i}=\ln \frac{r_{i}}{r_{c}} ; t_{j}=j \tau ; i=0,1,2, \ldots, N ; j=0,1,2, \ldots\right\}$ is found in the form of iteration:

$$
\begin{gathered}
p_{i-1}^{j+1, v}=A_{i-1}^{j+1, v} p_{i}^{j+1, v}+C_{i-1}^{j+1, v}, \\
i=N-1, \ldots, 1, j=0,1,2, \ldots, v=1,2, \ldots \\
\sigma_{i}^{j+1, v}=\sigma_{i}^{j}+H_{1 i}^{j+1, v}+H_{2 i}^{j+1, v}-\tau \frac{D_{4 i}^{j}}{E_{2 i}^{j}}, \\
\text { where } \begin{array}{c}
A_{i}^{j+1, v}=\frac{\Psi_{1 i}^{j+1, v}+\Psi_{2 i}^{j+1, v}}{\Psi_{5 i}^{j+1, v}+\Psi_{6 i}^{j+1, v}+D_{1 i}^{j}} \\
C_{i}^{j+1, v}=\frac{\left(\Psi_{3 i}^{j+1, v}+\Psi_{4 i}^{j+1, v}\right) C_{i-1}^{j+1, v}-\tau E_{1 i}^{j}+D_{1 i}^{j} p_{i}^{j}}{\Psi_{5 i}^{j+1, v}+\Psi_{6 i}^{j+1, v}+D_{1 i}^{j}} \\
i=1,2, . ., N-1 \\
A_{0}^{j+1, v}=1, C_{0}^{j+1, v}=-\frac{q_{o i l}^{* j+1} \cdot \Delta \xi}{F_{o i l 0}^{j+1, v-1}}
\end{array}
\end{gathered}
$$




$$
\begin{aligned}
& \sigma_{0}^{j+1, v}=\sigma_{0}^{j}+G_{1}^{j+1, v}+G_{2}^{j+1, v}-\tau \frac{D_{40}^{j}}{E_{20}^{j}} \\
& \Psi_{4 i}^{j+1, v}=a_{2} \frac{e^{-2 \xi_{i}} K_{0} \tau}{(\Delta \xi)^{2}} \Phi_{g a s, i-1 / 2}^{j+1, v-1} \\
& \Psi_{1 i}^{j+1, v}=\frac{e^{-2 \xi_{i}} K_{0} \tau}{(\Delta \xi)^{2}} F_{i}^{j} \Phi_{o i l, i+1 / 2}^{j+1, v-1}, \\
& \Psi_{5 i}^{j+1, v}=\frac{e^{-2 \xi_{i}} K_{0} \tau}{(\Delta \xi)^{2}} F_{i}^{j}\left(\Phi_{o i l, i+1 / 2}^{j+1, v-1}+\left(1-A_{i-1}^{j+1, v}\right) \Phi_{o i l, i-1 / 2}^{j+1, v-1}\right) \\
& \Psi_{2 i}^{j+1, v}=a_{2} \frac{e^{-2 \xi_{i}} K_{0} \tau}{(\Delta \xi)^{2}} \Phi_{g a s, i+1 / 2}^{j+1, v-1}, \\
& \Psi_{6 i}^{j+1, v}=a_{2} \frac{e^{-2 \xi_{i}} K_{0} \tau}{(\Delta \xi)^{2}}\left(\Phi_{g a s, i+1 / 2}^{j+1, v-1}+\left(1-A_{i-1}^{j+1, v}\right) \Phi_{\text {gas }, i-1 / 2}^{j+1, v-1}\right) \\
& \Psi_{3 i}^{j+1, v}=\frac{e^{-2 \xi_{i}} K_{0} \tau}{(\Delta \xi)^{2}} F_{i}^{j} \Phi_{o i l, i-1 / 2}^{j+1, v-1}, \\
& D_{1 i}^{j}=\left(s^{\prime}\left(p_{i}^{j}\right)-\frac{\beta}{p_{a t}}\left(a\left(p_{i}^{j}\right)+p_{i}^{j} a\left(p_{i}^{j}\right)\right) \frac{m_{i}^{j} \sigma_{i}^{j}}{a\left(p_{i}^{j}\right)}+\frac{\beta}{p_{a t}}\left(m_{i}^{j}+m_{0} \beta_{r} p_{i}^{j}\right),\right. \\
& D_{2 i}^{j}=a_{1}+\frac{m_{i}^{j} \sigma_{i}^{j}}{a^{2}\left(p_{i}^{j}\right)} a^{\prime}\left(p_{i}^{j}\right) \frac{F_{i}^{j}}{D_{1 i}^{j}}, D_{3 i}^{j}=a_{2} \frac{m_{i}^{j} \sigma_{i}^{j}}{a^{2}\left(p_{i}^{j}\right)} a^{\prime}\left(p_{i}^{j}\right) \frac{1}{D_{1 i}^{j}}, \\
& D_{4 i}^{j}=\left(\frac{\sigma_{i}^{j}}{a\left(p_{i}^{j}\right)}+\frac{\beta}{p_{a m}} \cdot \frac{m_{i}^{j} \sigma_{i}^{j} a^{\prime}\left(p_{i}^{j}\right)}{a^{2}\left(p_{i}^{j}\right)} \frac{p_{i}^{j}}{D_{1 i}^{j}}\right)\left(\left(m_{0}\left(m_{1}+\gamma_{m} \beta_{r}\right)\left(p_{i}^{j}-p_{0}\right)+\gamma_{m}\left(m_{0}-m_{i}^{j}\right)\right)-m_{0} \beta_{r} \frac{E_{1 i}^{j}}{D_{1 i}^{j}}\right) \\
& H_{1 i}^{j+1, v}=\frac{D_{2 i}^{j}}{E_{2 i}^{j}} \frac{e^{-2 \xi_{i}} K_{0} \tau}{(\Delta \xi)^{2}}\left(\Phi_{o i l, i+1 / 2}^{* j+1, v}\left(p_{i+1}^{j+1, v}-p_{i}^{j+1, v}\right)-\Phi_{o i l, i-1 / 2}^{* j+1, v}\left(p_{i}^{j+1, v}-p_{i-1}^{j+1, v}\right)\right), \\
& H_{2 i}^{j+1, v}=\frac{D_{3 i}^{j}}{E_{2 i}^{j}} \frac{e^{-2 \xi_{i}} K_{0} \tau}{(\Delta \xi)^{2}}\left(\Phi_{\text {gas }, i+1 / 2}^{* j+1, v}\left(p_{i+1}^{j+1, v}-p_{i}^{j+1, v}\right)-\Phi_{g a s, i-1 / 2}^{* j+1, v}\left(p_{i}^{j+1, v}-p_{i-1}^{j+1, v}\right)\right), \\
& G_{1}^{j+1, v}=\left(\frac{K_{0} \tau}{(\Delta \xi)^{2}} \frac{\bar{k}_{o i l}\left(\sigma_{1}^{j+1, \nu-1}\right)}{\mu_{n}\left(p_{1}^{j+1, v}\right) a\left(p_{1}^{j+1, v}\right)}\left(p_{2}^{j+1, v}-p_{1}^{j+1, v}\right)-\frac{K_{0} \tau}{\Delta \xi} q_{o i l}^{{ }^{*}+1}\right) e^{-2 \xi_{1}} \frac{D_{20}^{j}}{E_{20}^{j}}, \\
& G_{2}^{j+1, v}=\left(\frac{\bar{k}_{g a s}\left(\sigma_{1}^{j+1, v-1}\right)}{\mu_{g a s}\left(p_{1}^{j+1, v}\right)}\left(p_{2}^{j+1, v}-p_{1}^{j+1, v}\right)-\frac{\bar{k}_{\text {gas }}\left(\sigma_{0}^{j+1, v-1}\right)}{\mu_{\text {gas }}\left(p_{0}^{j+1, v}\right)}\left(p_{1}^{j+1, v}-p_{0}^{j+1, v}\right)\right) \frac{\tau K_{0} e^{-2 \xi_{1}}}{(\Delta \xi)^{2}} \frac{D_{30}^{j}}{E_{20}^{j}} \\
& E_{1 i}^{j}=\frac{\beta}{p_{a t}} p_{i}^{j}\left[m_{0}\left(m_{1}+\gamma_{m} \beta_{r}\right)\left(p_{i}^{j}-p_{0}\right)+\gamma_{m}\left(m_{0}-m_{i}^{j}\right)\right], E_{2 i}^{j}=\frac{m_{i}^{j}}{a\left(p_{i}^{j}\right)} \\
& \Phi_{o i l, i \pm 1 / 2}^{* j+1, v}=\frac{2 F_{o i l, i}^{* j+1, v} F_{o i l, i \pm 1}^{* j+1, v}}{F_{o i l, i}^{* j+1, v}+F_{o i l, i \pm 1}^{* j+1, v}}, \Phi_{\text {gas }, i \pm 1 / 2}^{* j+1, v}=\frac{2 F_{\text {gas }, i}^{* j+1, v} F_{\text {gas }, i \pm 1}^{* j+1, v}}{F_{g a s, i}^{* j+1, v}+F_{\text {gas }, i \pm 1}^{* j+1, v}} \\
& F_{o i l, i}^{* j+1, v}=\frac{\bar{k}_{o i l}\left(\sigma_{i}^{j+1, v-1}\right)}{\mu_{o i l}\left(p_{i}^{j+1, v}\right) \cdot a\left(p_{i}^{j+1, v}\right)} \\
& F_{g a s, i}^{* j+1, v}=\frac{\bar{k}_{g a s}\left(\sigma_{i}^{j+1, v-1}\right) \cdot p_{i}^{j+1, v}}{\mu_{g a s}\left(p_{i}^{j+1, v}\right) \cdot a\left(p_{i}^{j+1, v}\right)}+\frac{p_{a t}}{\beta} \frac{\bar{k}_{o i l}\left(\sigma_{i}^{j+1, v-1}\right) \cdot s\left(p_{i}^{j+1, v}\right)}{\mu_{o i l}\left(p_{i}^{j+1, v}\right) \cdot a\left(p_{i}^{j+1, v}\right)} \\
& F_{o i l, i \pm 1}^{* j+1, v}=\frac{\bar{k}_{o i l}\left(\sigma_{i \pm 1}^{j+1, v-1}\right)}{\mu_{o i l}\left(p_{i \pm 1}^{j+1, v}\right) \cdot a\left(p_{i \pm 1}^{j+1, v}\right)} \\
& F_{g a s, i \pm 1}^{* j+1, v}=\frac{\bar{k}_{g a s}\left(\sigma_{i \pm 1}^{j+1, v-1}\right) \cdot p_{i \pm 1}^{j+1, v}}{\mu_{g a s}\left(p_{i \pm 1}^{j+1, v}\right) \cdot a\left(p_{i \pm 1}^{j+1, v}\right)}+\frac{p_{a t}}{\beta} \frac{\bar{k}_{o i l}\left(\sigma_{i \pm 1}^{j+1, v-1}\right) \cdot s\left(p_{i \pm 1}^{j+1, v}\right)}{\mu_{o i l}\left(p_{i \pm 1}^{j+1, v}\right) \cdot a\left(p_{i \pm 1}^{j+1, v}\right)}
\end{aligned}
$$




$$
\begin{aligned}
& \Phi_{o i l, i \pm 1 / 2}^{j+1, v}=\frac{2 F_{o i l, i}^{j+1, v} F_{o i l, i \pm 1}^{j+1, v}}{F_{o i l, i}^{j+1, v}+F_{o i l, i \pm 1}^{j+1, v}}, \Phi_{g a s, i \pm 1 / 2}^{j+1, v}=\frac{2 F_{g a s, i}^{j+1, v} F_{g a s, i \pm 1}^{j+1, v}}{F_{g a s, i}^{j+1, v}+F_{g a s, i \pm 1}^{j+1, v}} \\
& F_{o i l, i}^{j+1, v}=\frac{\bar{k}_{o i l}\left(\sigma_{i}^{j+1, v}\right)}{\mu_{o i l}\left(p_{i}^{j+1, v}\right) \cdot a\left(p_{i}^{j+1, v}\right)}, \\
& F_{\text {gas }, i}^{j+1, v}=\frac{\bar{k}_{\text {gas }}\left(\sigma_{i}^{j+1, v}\right) \cdot p_{i}^{j+1, v}}{\mu_{\text {gas }}\left(p_{i}^{j, k}\right) \cdot a\left(p_{i}^{j+1, v}\right)}+\frac{p_{a t}}{\beta} \frac{\bar{k}_{\text {oil }}\left(\sigma_{i}^{j+1, v}\right) \cdot s\left(p_{i}^{j+1, v}\right)}{\mu_{\text {oil }}\left(p_{i}^{j+1, v}\right) \cdot a\left(p_{i}^{j+1, v}\right)}, \\
& F_{o i l, i \pm 1}^{j+1, v}=\frac{\bar{k}_{o i l}\left(\sigma_{i \pm 1}^{j+1, v}\right)}{\mu_{o i l}\left(p_{i \pm 1}^{j+1, v}\right) \cdot a\left(p_{i \pm 1}^{j+1, v}\right)}, \\
& F_{\text {gas }, i \pm 1}^{j+1, v}=\frac{\bar{k}_{\text {gas }}\left(\sigma_{i \pm 1}^{j+1, v}\right) \cdot p_{i \pm 1}^{j+1, v}}{\mu_{\text {gas }}\left(p_{i \pm 1}^{j+1, v}\right) \cdot a\left(p_{i \pm 1}^{j+1, v}\right)}+\frac{p_{a t}}{\beta} \frac{\bar{k}_{\text {oil }}\left(\sigma_{i \pm 1}^{j+1, v}\right) \cdot s\left(p_{i \pm 1}^{j+1, v}\right)}{\mu_{\text {oil }}\left(p_{i \pm 1}^{j+1, v}\right) \cdot a\left(p_{i \pm 1}^{j+1, v}\right)} \\
& F_{i}^{j}=a_{1}\left(\frac{\beta}{p_{a t}} p_{i}^{j} a\left(p_{i}^{j}\right)-s\left(p_{i}^{j}\right)\right), p_{N}^{j+1, v}=p_{N-1}^{j+1, v}=\frac{C_{N-1}^{j+1, v}}{1-A_{N-1}^{j+1, v}} \\
& q_{o i l}^{* j+1}=\frac{q_{o i l}^{j+1}}{2 \pi K_{0} h} ; a_{1}=\frac{1}{r_{w}^{2}} ; a_{2}=\frac{\beta}{r_{w}^{2} p_{a t}} ; p_{i}^{0}=p_{0} ; m_{i}^{0}=m_{0} ; \sigma_{i}^{0}=1 .
\end{aligned}
$$

In this iterative process internal iterations for porosity are carried out thus:

$$
m_{i}^{j+1}=m_{i}^{j}+m_{0} \beta_{s}\left(p_{i}^{j+1, \nu}-p_{i}^{j}\right)+m_{0} \tau\left(m_{1}+\gamma_{m} \beta_{s}\right)\left(p_{i}^{j}-p_{0}\right)+\gamma_{m} \tau\left(m_{0}-m_{i}^{j}\right)
$$

In each cycle of iterations at $v=1$ is accepted that

$$
p_{i}^{j+1,0}=p_{i}^{j}, \sigma_{i}^{j+1,0}=\sigma_{i}^{j}, m_{i}^{j+1,0}=m_{i}^{j} .
$$

For each temporary layer of $j+1$ at all $i$ of iteration proceed before performance of an inequality

$$
\max _{i}\left|p_{i}^{j+1, v}-p_{i}^{j+1, v-1}\right| \leq \varepsilon(v=1,2, \ldots)
$$

where $v$ - number of iteration; $\varepsilon$ - admissible error. After determination of values $p_{i}^{j+1}$ the corresponding values of porosity $m_{i}^{j+1}$ are defined thus:

$$
m_{i}^{j+1}=m_{i}^{j}+m_{0} \beta_{s}\left(p_{i}^{j+1}-p_{i}^{j}\right)+m_{0} \tau\left(m_{1}+\gamma_{m} \beta_{R}\right)\left(p_{i}^{j}-p_{0}\right)+\gamma_{m} \tau\left(m_{0}-m_{i}^{j}\right)
$$

We will note that from this iterative process it is possible to receive the corresponding iterative process for a case linearly - elastic deformation of breeds of layer. For this purpose, it is enough in formulas (13) and (15) of values of coefficients $m_{1}$ and $\gamma_{m}$ to equate to zero.

\section{Numerical Experiment and Analysis of Results}

In compliance of the specified iterative process (12-15), at the set output of a oil well changes in time and are determined by radial coordinate reservoir pressure, oil saturation and porosity of layer, including, changes in time of their bottom-hole and boundary values. Also change of a gas- oil ratio in time is defined.

Calculations were carried out on the basis of the following basic data:

$$
\begin{gathered}
p_{0}=40 \mathrm{MPa} ; q_{\text {oil }}=150 \mathrm{~m}^{3} / \text { days } ; m_{0}=0,2 ; \\
r_{w}=0,1 \mathrm{~m} ; r_{k}=300 \mathrm{~m} ; \beta=0,735 ; \\
z\left(p_{0}\right)=1,0385 ; p_{a t}=0,1 \mathrm{MPa} ; \beta_{r}=2,5 \cdot 10^{-3} \mathrm{MPa}^{-1} ; \\
K_{0}=0,1 \cdot 10^{-12} \mathrm{~m}^{2 ;} m_{1}=3,4 \cdot 10^{-8}(\mathrm{MPa} \cdot \mathrm{sec})^{-1} ; \gamma_{m}=4,3 \cdot 10^{-6} \\
\sec ^{-1} ; \mu_{\text {oil }}=\mu_{\text {oil } 0} \exp \left(-0,005\left(p-p_{0}\right)\right) ; \\
\mu_{\text {oil } 0}=0,7 \mathrm{mPa} \cdot \mathrm{sec} ;
\end{gathered}
$$




$$
\begin{gathered}
\bar{k}_{\text {oil }}=1,06 \cdot \sigma^{3}-0,06 ; \bar{k}_{\text {gas }}=1,16(1-\sigma)^{2} ; \\
\mu_{\text {gas }}(p)=0,0054\left(p / p_{0}\right)^{2}+0,0114\left(p / p_{0}\right)+0,0105, \\
\mathrm{mPa} \cdot \mathrm{sec} ; \\
s(p)= \begin{cases}3,9 \cdot p+15,5, & p \geq 5 \mathrm{MPa} \\
4 \cdot p, & p<5 \mathrm{MPa}\end{cases} \\
a(p)= \begin{cases}0,0058 \cdot p+1,021, & p \geq 5 \mathrm{MPa} \\
0,01 \cdot p+1, & p<5 \mathrm{MPa}\end{cases}
\end{gathered}
$$

Some results of calculations are presented in Figures 1-4. Here is illustrated only the results of determination of dynamic characteristics of reservoir development relative to change in time, however, the results obtained for the change of these indicators on the radial coordinate.

Analysis of the results shows that, in the present case the task of well production influence of creeping deformation of rocks of the reservoir in the determined parameters is large enough relative to their nonlinear elastic deformation.

The change in the radial coordinate manifests itself especially in a small neighborhood of the wells, and around the radial coordinate of character pattern impact time of creeping deformation of rocks on the dynamic characteristics of reservoir development (filtration flow) is the same way.

Relative to the nonlinear-elastic deformation of rocks creeping deformation in nature in different ways to affect the determined parameters. So while creeping deformation of rocks, the reduction in bottomhole pressure, oil saturation and gas factor is highly elabieta, but on the contrary, the values of porosity has a great temp decrease. The difference in the values of the defined indicators in each case, the deformability of the rock of the reservoir over time to become even more significant.

The proposed design algorithm may be implemented in the calculations to determine the dynamic characteristics of the filtration of carbonated oil, manifested itself, during the development of oil fields in the depletion mode to reflect the impact of the creeping deformation of rocks on these characteristics, as well as ensure prompt diagnosis of the reservoir system in the manifestation in it of rheological deformations like creep of rocks.

Rather nonlinear and elastic deformation of rocks of a collector creeping deformation of rocks on character variously influences the defined indicators. So at creeping deformation of rocks decrease in bottom-hole pressure, oil saturation and a gas factor is strongly weakened, and on the contrary, values of porosity big speed decrease possesses. Distinction in values of the defined indicators in each case of deformability of rock of layer is getting still considerable over time.

The offered settlement algorithm can is carried out when calculating for definition of dynamic characteristics of a filtration of the aerated oil which is proved during development of oil pools in the exhaustion mode for the purpose of the account influence of creeping deformation of rocks on these characteristics and also for the purpose of expeditious diagnosing of sheeted system at manifestation in him rheological deformations as creep of rocks.

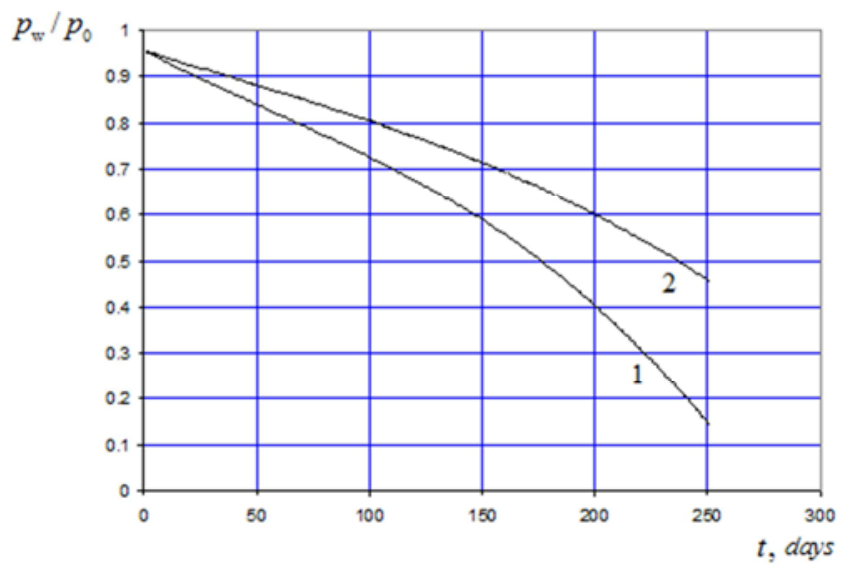

Figure 1. Change of bottom-hole pressure in time.

1 - nonlinear - elastic deformation

2 - creeping deformation

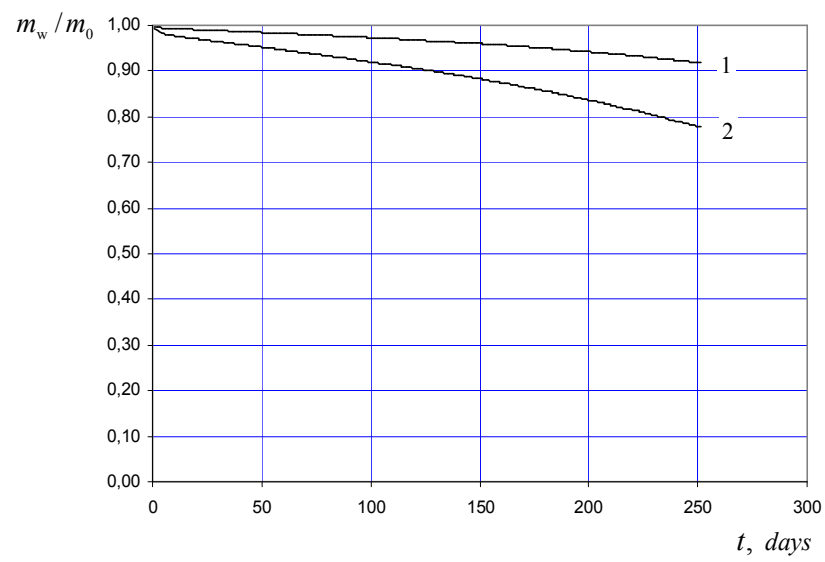

Figure 2. Change of porosity in time.

1 - nonlinear - elastic deformation

2 - creeping deformation

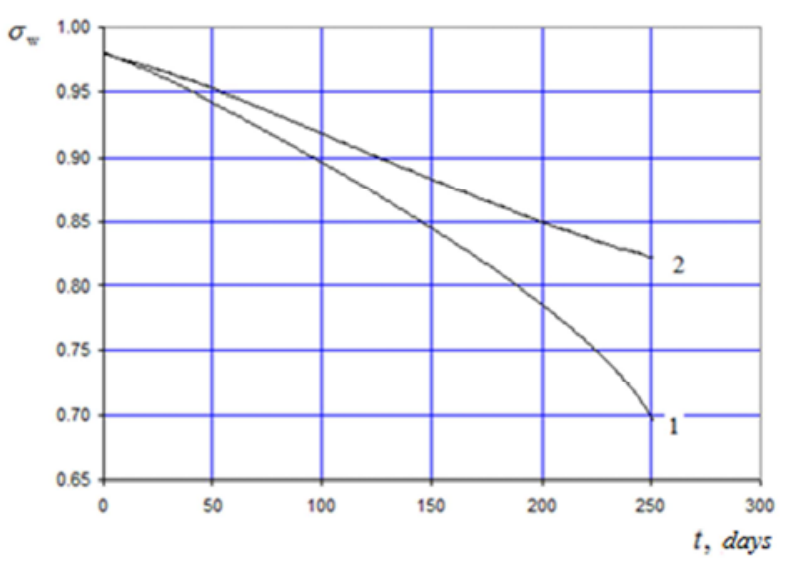

Figure 3. Change of oil saturation in time.

1 - nonlinear - elastic deformation

2 - creeping deformation 


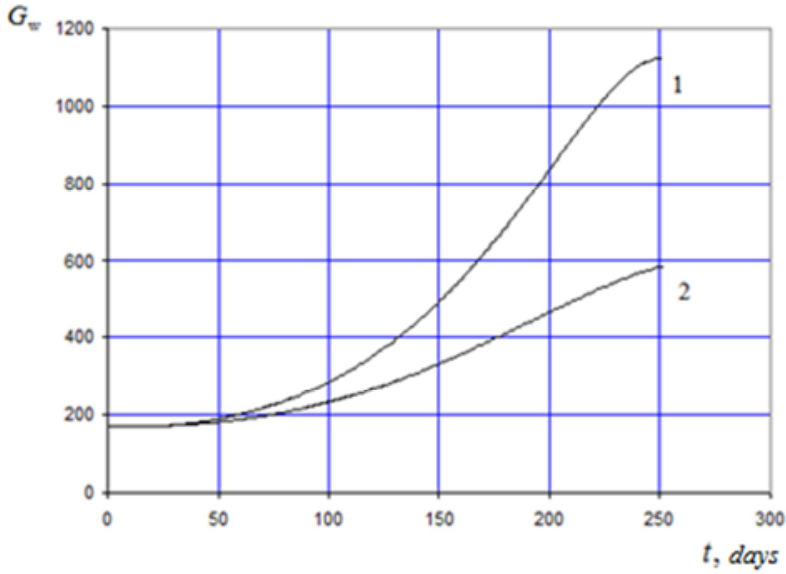

Figure 4. Change of a gas factor in time.

1 - nonlinear - elastic deformation

2 - creeping deformation

\section{Conclusion}

Is provided the numerical solution of a problem of a filtration of gas-cut oil to a well in creeping layer. On the basis of the proposed numerical solution, changes of indicators of the development (reservoir pressure, porosity, gas-oil ratio and etc.) can be determined in time and by radial coordinate in oil layer which breeds are exposed to creeping deformations. The offered algorithm can be successfully applied when calculating for accounting of creep of rocks in case of determination of the current values of the main dynamic characteristics of filtering the aerated oil which is proved during development of oil fields in the depletion mode.

\section{Additional Notes}

Article is prepared within the research program of National Academy of Sciences of Azerbaijan for the subject "Complex theoretical and experimental studies of interdisciplinary problems of geomechanics" approved by the Resolution of Presidium of NASA No. 5/3 of February 11, 2015, (2015 2017).

\section{References}

[1] I. M. Ametov, I. M. Basniyev, Filtration of liquid and gas in creeping environments. News of Academy of Sciences of the USSR, ser. Mechanics of liquid and gas, 4, 1981, pp.150-153.

[2] Yu. M. Molokovich, A no-equilibrium filtration and its application in oil-field practice, Sentrlitneftgaz: Moscow, 2006, $214 \mathrm{p}$.

[3] A. M. Guliyev, B. Z. Kazimov, Deformation of rocks and its influence on their filtrational and capacitor properties and on processes of a filtration and development of deposits of oil and gas, Elm: Baku, 2009, 88 p.

[4] A. M Guliyev, B. Z. Kazymov, Study of rhealogical properties of reservoirs during relaxation and creep strain of rocks and usage of these properties for addressing of applied objectives of oil-gas fields development, Special Issue Papers of 34th International Geological Congress "The modern problems of geology and geophysics of Eastern Caucasus and the South Caspian depression”, Nafta-Press: Baku, 2012, p. 117-131.

[5] M. A. Dunyamalyev, A. M. Guliyev, The methodical guide to determination of filtration and rheological properties of layer according to restoration of bottom-hole pressure of wells, AzTU: Baku, 1990, 63 p.

[6] M. T. Abasov, Z. A. Kerimov, D. R. Mirzoyeva, T. Sh. Kazymova, About determination of parameters of the creeping and compressed layers according to hydrodynamic researches of relaxation wells, News of NAS ofAzerbaijan, ser. Sciences about the Earth,1, 2006, pp. 59-64.

[7] T. Sh. Kazymova, About determination of parameters of creeping layer according to hydrodynamic researches of wells, News NAS ofAzerbaijan, ser. Sciences about the Earth, 1, 2008, pp. 112-117.

[8] Kh. A. Feyzullayev, Improving the simulation of water and gas flows foundations of the development of deep gas condensate fields, Dis. on competition of a scientific degree. doc. tech. sciences. NASA IG, 2011, 303 p.

[9] S. N. Zakirov, Development gas, gas-condensate and oil-gascondensate fields, String: Moscow, 1998, 628 p.

[10] X, Aziz, E. Settari, Mathematical modeling of sheeted systems: translation from English, Nedra: Moscow, 1982, 407 p. 